Ann. Génét. Sél. anim., 1976, 8 (4), 527-535.

\title{
GAIN DE POIDS PENDANT LA PONTE ET SÉLECTION SUR L'EFFICACITÉ ALIMENTAIRE CHEZ LA POULE
}

\author{
A. BORDAS et P. MÉRAT \\ Laboratoive de Génétique factorielle, \\ Centre national de Recherches zootechniques, I. N. R. A., \\ 78350 Jouy-en-Josas

\section{RÉSUMÉ}

La variation du poids corporel de pondeuses en cages a été mesurée d'une part entre les âges de 8 et $I x$ mois sur des poules dont la consommation d'aliment était enregistrée, d'autre part entre les âges de I 7 semaines et Io mois sur d'autres poules. Dans chaque groupe, une partie des poules était de génotype $D w$, les autres étaient naines (dw).

Dans les deux cas l'héritabilité du critère étudié est appréciable, suggérant la possibilité d'une sélection.

Le premier critère présente chez les poules Dw une corrélation positive relativement élevée avec la consommation alimentaire à poids corporel et poids total d'œufs pondus fixés, ainsi qu'une corrélation négative avec le nombre d'œufs et la masse totale d'œufs pondus. La réduction par sélection du gain de poids ainsi défini pourrait donc améliorer le rendement alimentaire des pondeuses sans réponses liées défavorables par ailleurs. La situation est analogue chez les poules naines, sauf la possibilité d'une réponse liée défavorable sur le poids moyen des œufs.

Quant à la variation de poids entre les âges de 17 semaines et de Io mois, sa réduction éventuelle par sélection paraît moins avantageuse chez les poules Dw d'après les corrélations observées (risque d'une điminution du poids moyen des œufs) et franchement défavorable chez les naines (risque d'abaissement du nombre et du poids des œufs).

\section{INTRODUCTION}

L'efficacité de la conversion de l'aliment par les poules pondeuses dépend pour une large part de leur ponte et de leur poids corporel, secondairement de leur variation de poids pendant la période étudiée. Ces facteurs sont mis en évidence, sous forme d'une équation de régression multiple, par BYERLY (I94I) et d'autres auteurs, par exemple LEEESON et al. (I973).

Par sélection augmentant le taux de ponte et diminuant le poids des poules adultes, des gains importants ont été réalisés sur le rendement alimentaire des lignées et croisements spécialisés pour la ponte. Il peut d'autre part subsister, à 
poids corporel, gain de poids et production d'œufs égaux, une variabilité " résiduelle " de la consommation d'aliment, correspondant à des différences dans l'ingestion et l'efficacité d'utilisation des nutriments ingérés : nous avons montré (BORDAs et MÉRAT, I974) que cette variabilité peut avoir une composante génétique permettant d'envisager une sélection soit directe, relativement laborieuse, soit indirecte, de portée plus limitée mais simple si elle utilise par exemple des critères morphologiques.

D'autre part, il ne semble pas que la variation de poids durant la ponte, pourtant facile à mesurer, ait été utilisée comme critère complémentaire de sélection pour le rendement alimentaire. Nous avons voulu faire, à partir de données de consommation individuelles de poules en ponte recueillies depuis 1967 , une première évaluation des possibilités dans ce sens.

\section{MATÉRIEL, ET MÉTHODES}

\section{I. - Animaux contrôlés individuellement pour leur consommation alimentaire}

Les données sont celles mentionnées dans notre article đéjà cité (Bordas et MÉrat, 1974), utilisées dans une optique différente, et avec l'addition de deux années supplémentaires. Nous en rappellerons seulement les caractéristiques principales.

Une première série de lots expérimentaux, de 1967 à 1975, est issue de la population élevée à Jouy-en-Josas, comportant une ségrégation à divers loci connus. Un second groupe, de I97 I à 1975, comprend des échantillons d'une souche " ponte "Rhode-Island rouge élevée à Jouy, issue à l'origine de la Station expérimentale du Magneraud (souche M 99). Enfin, dans la population de Jouy, nous consiaérons à part, en 1973 et 1974, des poules issues d'une partie des familles mais possédant le gène de nanisme lié au sexe dw.

Pour tous ces lots, les jeunes sont élevés au sol jusqu'à l'âge de 16 semaines, puis mis en cages individuelles. L'enregistrement de la consommation alimentaire commence à l'âge de 8 mois environ et se poursuit pendant trois périodes consécutives de 28 jours. L'aliment, de formule identique d'une année sur l'autre, contient $\mathrm{x} 6 \mathrm{p}$. cent de protéines et $2520 \mathrm{kcal}$ d'énergie métabolisable par kg, sous forme de farine, sauf en I970 et I97I (granulés).

Par animal et par période sont enregistrés la consommation alimentaire $O$, le poids corporel moyen $\mathrm{P}$, la variation $\Delta \mathrm{P}$ du poids du début à la fin de la période (les animaux étant mis à jeun la veille au soir de chaque pesée qui avait lieu le matin, et sans par ailleurs faire de correction pour les pontes ayant eu lieu le jour des pesées); enfin le poids total des œufs pondus E. Seules sont considérées les poules ayant eu toutes les mesures durant les trois périodes. Les seuls résultats utilisés ici portent sur la moyenne des trois périodes pour chaque variable.

La consommation " théorique " $T$ par individu est estimée en fonction des variables précédentes par une équation de régression multiple (BYERLY, I94I) du type

$$
\mathrm{T}=a \mathrm{P}^{\alpha}+b \Delta \mathrm{P}+c \mathrm{E}
$$

Les performances moyennes des animauxétudiés (Poids corporel, ponte) ont été mentionnées dans notre précédent article (BORDAS et MERAT, 1974).

Nous nous intéressons ici plus spécialement à la variable $\Delta P$, dans ses corrélations avec d'autres variables représentatives du taux de ponte, des caractéristiques des œufs, de la croissance et du poids adulte, variables définies dans le tableau I.

\section{2. - Troupeau non contrôlé individuellement pour la consommation alimentaire}

Sur plusieurs générations du troupeau expérimental de Jouy, entre 1972 et 1975 (non compris l'échantillon dont la consommation alimentaire était contrôlée) deux pesées de chaque individu ont été faites, à I 7 semaines d'âge (avant les premières entrées en ponte) puis approximativement à ro mois d'âge; la différence entre la seconde et la première est appelée a variation de poids ». Une partie des animaux était éclose au printemps (en 1974 et 1975); deux autres générations (1972 et 1974), issues d'une fraction distincte du troupeau, étaient nées à l'automne et incluaient dans une partie des familles des poules naines (dw). 
L'héritabilité du facteur a variation de poids » a été estimée uniquement sur les deux générations nées au printemps et comportant des poules toutes de génotype $\mathrm{Dw}$.

Par ailleurs, des corrélations ont été estimées entre cette variation de poids et d'autres variables quantitatives, mentionnées dans le tableau correspondant (tabl. 3).

\section{RÉSULTTATS}

\section{TABLEAU I}

Corrélations entre la variation de poids de poules en ponte (sur 3 périodes de 28 jours entre les âges de 8 et II mois) et d'autres caracteres quantitatifs

(poules contrôlées pour la consommation d'aliment)

Correlations between body weight variation of layers (over 3 periods of 28 days between the ages 8 and II months) and otheriquantitative traits

(hens with recorded feed consumption)

\begin{tabular}{|c|c|c|c|c|c|c|}
\hline \multirow{3}{*}{$2^{\mathrm{e}}$ caractère } & \multicolumn{6}{|c|}{ Corrélation } \\
\hline & \multicolumn{2}{|c|}{ Poules Dw } & \multicolumn{2}{|c|}{ Poules dw } & \multicolumn{2}{|c|}{ Total } \\
\hline & d. 1. & $\mathbf{r}$ & d. 1 . & $\mathbf{r}$ & d. 1 . & $\mathbf{r}$ \\
\hline $\begin{array}{c}\mathrm{O}=\begin{array}{c}\text { Consommation observée } \\
\text { (Observed feed intake) }\end{array}\end{array}$ & 869 & $+0,39^{* * *}$ & 81 & $+0,43^{* * *}$ & 950 & $+0,39^{* * *}$ \\
\hline 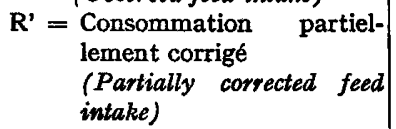 & 869 & $+0,52^{* * *}$ & 81 & $+0,50^{* * *}$ & 950 & $+0,52^{* * *}$ \\
\hline $\begin{array}{l}\text { Consommation d'eau } \\
\text { (Water intake) }\end{array}$ & 731 & $+0,05$ & 73 & $+0,36 * *$ & 804 & $+0,08$ \\
\hline $\begin{array}{l}\mathrm{E}=\text { Poids total d'œufs pondus } \\
\text { (Total weight of eggs) }\end{array}$ & 869 & $-0,15^{* * *}$ & 81 & $-0,12$ & 950 & $\longrightarrow 0,15^{* * *}$ \\
\hline $\begin{array}{l}\text { Age au } 1^{\text {er }} \text { œuf } \\
\left.\text { (Age at } 1^{\text {st }} \mathrm{egg}\right)\end{array}$ & 746 & $-0,10^{* *}$ & 73 & $-0,18$ & 819 & $-0,11^{* *}$ \\
\hline $\begin{array}{l}\text { Nombre d'œufs } \\
\text { (Egg number) }\end{array}$ & 834 & $-0,13^{* *}$ & 80 & $-0,06$ & 914 & $-0,12^{* *}$ \\
\hline $\begin{array}{l}\text { Poids moyen des œufs } \\
\text { (Mean egg weight) }\end{array}$ & 834 & $+0,06$ & 80 & $+0,27^{*}$ & 914 & $+0.08^{*}$ \\
\hline $\begin{array}{l}\text { Hauteur d'albumen } \\
\text { (Albumen height) }\end{array}$ & 792 & $-0,02$ & 73 & $+0,12$ & 865 & $-0,01$ \\
\hline $\begin{array}{l}\text { Epaisseur de coquille } \\
\text { (Shell thickness) } \\
\text { Poids adulte (moyenne sur les }\end{array}$ & 753 & $-0,07$ & 73 & $-0,05$ & 826 & $-0,07$ \\
\hline $\begin{array}{l}3 \text { périodes) } \\
\text { (Adult weight, average over } 3 \\
\text { periods) }\end{array}$ & 869 & $+0,31^{* * *}$ & 81 & $+0,40^{* * *}$ & 950 & $+0,32 * * *$ \\
\hline $\begin{array}{l}\text { Longueur des barbillons } \\
\text { (Wattle length) }\end{array}$ & 799 & $-0,01$ & 73 & $+0,25^{*}$ & 872 & $+0,01$ \\
\hline $\begin{array}{l}\text { Longueur des tarses } \\
\text { (Shank length) }\end{array}$ & 799 & $+0,09^{*}$ & 73 & $+0,39^{* * *}$ & 872 & $+0,12^{* *}$ \\
\hline
\end{tabular}

* Significatif au seuil 5 p. Ioo.

* Significatif au seuil I p. 100.

*** Significatif au seuil $\mathrm{r}$ p. $\mathrm{r}$ 00o. 
TABLEAU 2

Héritabilité de la variation de poids entre 17 semaines et 10 mois d'âge (troupeau, années r974 et 1975)

Heritability of body weight variation between 17 weeks and 10 months of age (flock, years 1974 and 1975)

\begin{tabular}{c|c|c|c|c|c|c}
\hline \hline \multirow{2}{*}{ Année } & \multicolumn{3}{|c|}{ Effectifs } & $h^{2}$ & $h^{2}$ & $h^{2}$ \\
\cline { 2 - 6 } & Enfants & Mères & Pères & $d$ & & \\
\hline 1974 & 395 & 54 & 19 & 0,37 & 0,83 & 0,60 \\
1975 & 338 & 63 & 19 & 0,60 & 0,43 & 0,52 \\
\hline \hline
\end{tabular}

TABLEAU 3

Corrélation entre la variation de poids de 17 semaines à 10 mois d'âge et d'autres caractères quantitatifs

Correlation between body weight variation from 17 weeks to 10 months of age and other quantitative traits

\begin{tabular}{|c|c|c|c|c|c|c|}
\hline \multirow{2}{*}{$2^{e}$ caractère } & \multicolumn{2}{|c|}{$\begin{array}{l}\text { Poules normales } \\
\text { (Dw) }\end{array}$} & \multicolumn{2}{|c|}{$\begin{array}{l}\text { Naines } \\
\text { (dw) }\end{array}$} & \multicolumn{2}{|c|}{ Total } \\
\hline & d. 1 . & $\mathbf{r}$ & d. 1. & $\mathbf{r}$ & d. 1. & $r$ \\
\hline $\begin{array}{l}\text { Poids initial }(17 \mathrm{sem}) \\
\text { (Initial body weight) }\end{array}$ & 917 & 0,00 & 237 & $+0,08$ & 1154 & $+0,02$ \\
\hline $\begin{array}{l}\text { Age au } 1 \text { er cuf } \\
\text { ( } A \text { ge at } 1^{\text {st }} \text { egg) } \\
\text { Nombre d'œufs (entrée en ponte } \\
\text { jusqu'à } 10 \text { mois d'âge) }\end{array}$ & 898 & $+0,23^{* * *}$ & 237 & $+0,03$ & 1135 & $(+0,19 * * *)$ \\
\hline $\begin{array}{l}\text { jusqu'à } 10 \text { mois d'âge) } \\
\text { (Egg number, first egg to } \\
10 \text { mths age) }\end{array}$ & 898 & $-0,15^{* * *}$ & 237 & $+0,23^{* * *}$ & 1135 & $(-0,08)$ \\
\hline $\begin{array}{l}\text { Intensité de ponte } \% \text { (pauses } \\
\text { déduites) } \\
\text { (Laying intensity, } \\
\text { deduced) }\end{array}$ & 898 & $+0,02$ & 237 & $+0,25^{* * *}$ & 1135 & $(+0,07)$ \\
\hline $\begin{array}{l}\% \text { jours de pauses } \\
\text { (\% pause days) }\end{array}$ & 898 & $-0,02$ & 237 & $-0,05$ & 1135 & $-0,03$ \\
\hline $\begin{array}{l}\text { Poids moyen des œufs (à } 10 \text { mois) } \\
\text { (Mean egg weight at } 10 \text { mths) }\end{array}$ & 898 & $+0,27^{* * *}$ & 237 & $+0,32 * * *$ & 1135 & $+0,28 * * *$ \\
\hline $\begin{array}{l}\text { Hauteur albumen (a } 10 \text { mois) } \\
\text { (Albumen height) }\end{array}$ & 917 & $+0,06$ & 237 & $+0,03$ & 1154 & $+0,05$ \\
\hline $\begin{array}{l}\text { Épaisseur de coquille (à } 10 \text { mois) } \\
\text { (Shell thickness) }\end{array}$ & 917 & $+0,02$ & 237 & $-0,02$ & 1154 & $+0,01$ \\
\hline $\begin{array}{l}\text { Hématocrite (à } 10 \text { mois) } \\
\text { (Hematocrit) }\end{array}$ & 693 & $+0,08$ & 176 & $+0,32^{* * *}$ & 869 & $\left(+0,13^{* *}\right)$ \\
\hline
\end{tabular}

* Significatif au seuil 5 p. roo.

** Significatif au seuil I p. roo.

*** Significatif au seuil I p. I ooo.

Les corrélations figurant au tableau I se rapportent au premier ensemble de données (animaux en contrôle de consommation individuelle) et concernant la variation de poids en trois périodes successives de 28 jours. Le second caractère 
est défini dans la première colonne; sauf 1'âge au premier œuf, il concerne des mesures prises sur l'ensemble des trois périodes, ou en fin de dernière période (hauteur de l'albumen, épaisseur de coquille, longueur des barbillons et des tarses).

Les tableaux 2 et 3 , obtenus à partir du second ensemble de données (troupeau sans contrôle de corsommation alimentaire), concernent la variation de poids entre I7 semaines et Io mois d'âge. Le tableau 2 présente l'héritabilité estimée de cette variation de poids. Le tableau 3 montre des corrélations analogues à celles du tableau $I$.

En ce qui concerne les corrélations figurant tant au tableau I qu'au tableau 3, du fait de l'absence d'hétérogénéité entre lots et années, les évaluations de ces corrélations, faites sur une base intra-années, sont présentées regroupées pour toutes les années, pour toutes les poules normales (Dw) d'une part, pour les naines (dw) de l'autre. Dans le cas du tableau $x$, il n'apparaît pas non plus de différences significatives entre poules Dw et $\mathrm{dw}$ pour les diverses corrélations, dont nous donnons la valeur pour l'ensemble des deux génotypes. Pour le tableau 3, nous présentons également cette valeur, mais elle est indiquée entre parenthèses dans le cas où une différence significative $(P<0,0 x)$ apparaît entre poules normales et poules naines (corrélation de la variation de poids avec respectivement l'âge au $\mathrm{I}^{\mathrm{er}}$ œuf, le nombre d'œufs, l'intensité de ponte, 1'hématocrite).

\section{DISCUSSION ET CONCLUSIONS}

\section{I. - Signification et héritabilité des deux critères de variation de poids}

La variation de poids en 3 périodes de 28 jours se situe entre les âges approximatifs de 8 et II mois et nettement après l'entrée en ponte pour toutes les poules, cette dernière ayant lieu en majorité à l'âge de 5 à 6 mois ; cette variation de poids correspond vraisemblablement, pour une part importante, à un engraissement (LECLERCQ, I975). Nous avons antérieurement indiqué (BoRDAs et MÉRAT, I974) que ce critère présentait une variabilité appréciable entre familles de pères dans l'ensemble de nos résultats, correspondant à une héritabilité comprise entre 0,2 et 0,4 .

Pour la variation de poids enregistrée sur le reste du troupeau entre les âges de I7 semaines et de ro mois, elle correspond à une période plus longue, dont le début se place avant l'entrée en ponte (d'environ 2 semaines à plus de 2 mois suivant les individus). Le tableau 2 montre que l'héritabilité de cette variable est relativement élevée, apparemment plus que pour la précédente.

Les deux variables ayant été mesurées en général sur des séries différentes d'animaux, nous n'avons pu évaluer leur corrélation que sur une fraction restreinte - des données où elles se trouvaient enregistrées sur les mêmes oiseaux (I974 et I975). Sur I23 poules (Dw), le coefficient de corrélation correspondant est égal à $+0,62$. Sa valeur, significative $(P<0,0 r)$, suggère une liaison relativement forte entre ces variables.

\section{2. - Corrélations}

a) Le tableau I montre plusieurs corrélations phénotypiques intéressantes de la variation de poids $\Delta \mathrm{P}$ sur trois périodes de 28 jours. Les plus importantes sont 
celles avec la consommation observée $(\mathrm{O})$, et avec la consommation " partiellement corrigée " $\left(R^{\prime}\right)$. En termes de régression, on peut évaluer à environ I70 $\mathrm{g}$ la réduction de consommation d'aliment par 28 jours associée à une diminution de $100 \mathrm{~g}$ (soit $\mathrm{I}, 7$ écart-type) du gain de poids $\Delta \mathrm{P}$.

Sont appréciables également, quoique plus faibles, les corrélations de $\Delta \mathrm{P}$ avec le poids total d'œufs produits (E), le nombre d'œufs, 1'âge au I ${ }^{\text {er }}$ œuf et peutêtre le poids moyen des œufs. Les poules à plus faible gain de poids ont un poids total d'œufs pondus et un nombre d'œufs plus élevés en moyenne, quoique l'âge au Ier œuf soit légèrement plus tardif (en fixant cette dernière variable, la corrélation partielle entre $\Delta \mathrm{P}$ et le nombre d'œufs est égale à $-0,22$, supérieure en valeur absolue à la corrélation simple). Par ailleurs, le poids moyen de leurs œufs est un peu plus grand.

On peut remarquer qu'en enlevant sur l'ensemble de nos résultats un petit nombre d'animaux à variation de poids très négative, dont on peut se demander s'ils étaient dans un état de santé normal, la corrélation entre $\Delta \mathrm{P}$ et $\mathrm{E}$ est rendue encore plus négative, tous ces individus ayant une faible valeur pour $\mathrm{E}$.

Quant à la corrélation positive entre $\Delta \mathrm{P}$ et le poids adulte, elle s'explique en partie du fait que ce poids adulte est le poids moyen sur l'ensemble des 3 périodes de 28 jours considérées, et présente donc une corrélation automatique avec la variation de poids sur ces périodes. La corrélation de $\Delta \mathrm{P}$ avec le poids corporel initial (en début de première période) peut être estimée à $+0, \mathrm{I} 2(\mathrm{P}<0,0 \mathrm{r})$ sur l'ensemble des données pour les poules normales et à $+0,2 \mathrm{I}(\mathrm{P}<0,05)$ pour les naines. On peut vérifier grâce aux corrélations partielles que les corrélations de $\Delta \mathrm{P}$ avec le poids total des œufs ou leur nombre ne peuvent s'expliquer à partir de la corrélation de $\Delta \mathrm{P}$ avec le poids adulte, soit moyen soit initial.

Accessoirement, deux corrélations non directement perceptibles d'après le tableau I sont à noter. A poids corporel fixé, la corrélation partielle entre $\Delta \mathrm{P}$ et la longueur du tarse devient négative $(-0,34)$ chez les poules $D w$, ce qui peut simplement refléter une opposition automatique, pour un poids total donné, entre l'importance de différentes fractions de ce total (squelette et masses lipidiques).

D'autre part, à consommation d'aliment fixée, la corrélation partielle entre $\Delta \mathrm{P}$ et la consommation d'eau est, elle aussi, négative $(-0,3 \mathrm{r}$ chez les poules $\mathrm{Dw}$, $\mathbf{P}<0$, or); il en est de même à ponte fixée. Autrement dit, les poules à plus fort gain de poids consomment moins d'eau par rapport à la quantité d'aliment qu'elles ingèrent : ceci correspondrait à environ $50 \mathrm{ml}$ d'eau ingérée en moins pour roo $\mathrm{g}$ de gain de poids en plus. On pourrait penser à une plus faible rétention d'eau dans la mesure où un gain de poids plus élevé représente surtout une augmentation des masses lipidiques à teneur en eau inférieure à celle des autres tissus, mais il ne paraît pas certain que ceci rende compte de la totalité de la corrélation observée.

b) Le tableau 3 fait apparaître, en ce qui concerne la variation de poids entre les âges de 17 semaines et de 10 mois, des corrélations en partie différentes de celles du tableau I. Nous examinerons d'abord le cas des poules " normales ", Dw.

La corrélation avec le nombre d'œufs enregistré sur une période d'ailleurs plus longue reste négative et hautement significative.

Avec l'âge au $\mathrm{I}^{\mathrm{er}}$ œuf, le signe de la corrélation est inverse, puisque celle-ci est significativement positive : les poules à gain de poids le plus élevé sont celles, 
en moyenne, à entrée en ponte la plus tardive. Il ne s'agit pas de poules à plus faible poids à I7 semaines, comme le montre l'absence de corrélation entre ce poids et le gain de poids ultérieur, mais d'autre part, étant donnée la période considérée pour l'évaluation du gain de poids, ce dernier peut correspondre en partie au développement du tractus génital lors de l'entrée en ponte, ce qui peut suffire à expliquer la différence avec les données du tableau $\mathrm{I}$ dans le coefficient de corrélation en question.

D'autre part, au tableau 3, la corrélation de - 0,15 entre variation de poids et nombre d'œufs peut s'expliquer peut-être en totalité par celle entre cette variation et l'âge au I ${ }^{\text {er }}$ œuf, car la corrélation partielle variation de poids-nombre d'œufs à entrée en ponte fixée est égale à - 0,04, non significative.

La liaison entre le gain de poids et le poids moyen des œufs, quant à elle, est positive comme au tableau I, mais plus importante et hautement significative; ceci reste vrai à âge au I ${ }^{\text {er }}$ œuf fixé, ainsi qu'à poids corporel fixé.

Quant aux poules naines, elles présentent ici, comme il a été signalé plus haut, certaines valeurs de corrélations sensiblement différentes des normales; les naines à gain de poids élevé ont tendance à être les meilleures pondeuses, pour le nombre d'œufs et l'intensité de ponte. Précisément, chez ces poules, le gain de poids moyen pendant la ponte est inférieur à celui des poules Dw (MÉRAT et RICARD, 1974). D'ailleurs, il existe chez ce génotype une corrélation positive de l'ordre de $+0,4$ (MÉRAT, 1969) entre nombre d'œufs et poids adulte (vers ro mois), ce qui rend compte en partie de la corrélation observée.

Enfin, on constate chez les naines une corrélation positive, dont la signification reste à interpréter, entre le gain de poids et l'hématocrite.

Par contre, la corrélation positive entre gain de poids et poids moyen des œufs est analogue dans les deux génotypes $\mathrm{Dw}$ et $\mathrm{dw}$, de même que l'absence de liaison significative entre la première variable et le poids à $I 7$ semaines.

\section{3. - Utilisation possible des critères de variation de poids en sélection}

Il semble possible de modifier par sélection l'un ou l'autre des deux critères de variation de poids examinés ici, leur héritabilité apparaissant appréciable.

Les corrélations phénotypiques observées sont également, dans ce sens, une indication utile, dans la mesure où les corrélations génétiques seront de même ordre ou au moins de même sens.

a) Nous avons mentionné plus haut qu'une réduction de roo $\mathrm{g}$ du gain de poids entre 8 et 11 mois entraînait, d'après la valeur de la régression phénotypique, une diminution de consommation d'aliment d'environ $170 \mathrm{~g}$ par 28 jours dans nos conditions. Ceci représente de l'ordre de $5 \mathrm{p}$. roo de la consommation moyenne. Une diminution par sélection de ce gain de poids pourrait donc améliorer dans une mesure non négligeable le rendement alimentaire des pondeuses. Ceci n'est pas, ou n'est que partiellement, réalisé par la diminution, pratiquée en sélection " ponte ", du poids corporel à un âge donné, qui englobe le gain de poids antérieur. La régression de la consommation observée d'aliment sur les variables $E$ (poids d'œufs), $P$ (poids corporel) et $\Delta \mathrm{P}$, montre en effet, dans nos résultats en particulier (BoRDAS et MÉrAT, I974), qu'une variation de I $g$ sur $\Delta P$ produit sur la consommation alimentaire une variation environ double de celle accompagnant une variation de $\mathbf{I} \mathbf{g}$ portant sur $P$. D'autre part, on ne peut réduire indéfiniment le poids adulte total 
si l'on veut conserver un poids moyen des oufs suffisant; une sélection sur $\Delta P$ semble moins sujette à cet inconvénient.

Les corrélations du gain de poids de 8 à II mois avec le nombre et le poids des œufs suggèrent en effet, relativement à ces derniers caractères, la possibilité d'une réponse liée plutôt favorable à une sélection réduisant le gain de poids : diminution faible ou nulle du poids moyen des œufs, mais par contre un certain accroissement du nombre d'œufs, ainsi que la masse d'œufs produite. Il n'apparaît donc pas à cet égard de contre-indication à une telle sélection. Chez les poules $\mathrm{dw}$, toutefois, la possibilité d'une réponse liée sur le poids moyen des œufs plus défavorable que chez les normales est suggérée par nos données.

b) Quant à la variation de poids de 17 semaines à 10 mois d'age, nous n'avons estimé sa corrélation avec la consommation d'aliment (entre 8 et II mois d'âge) que sur I23 poules $\mathrm{Dw}$ (en I974 et I975). Au total, la valeur du coefficient de corrélation avec la consommation " partiellement corrigée " $R$ ' est égale à $+0,29(P<0,05)$, appréciable mais inférieure à celle obtenue au tableau $I$ avec la première évaluation du gain de poids.

Une sélection sur ce second critère de gain de poids pourrait donc permettre une certaine amélioration du rendement alimentaire des pondeuses, sans doute moins efficace qu'avec le premier. D'autre part, une réduction par sélection du gain de poids de I7 semaines à Io mois, de par les corrélations mentionnées au tableau 3 , risque d'entraîner chez les poules normales $\mathrm{Dw}$ une réponse liée favorable pour le nombre d'œufs pondus et la précocité sexuelle, mais par contre une réduction non négligeable du poids moyen des œufs. Par ailleurs, chez les poules naines dw, l'ensemble des réponses liées, pour le nombre et le poids des œufs, devrait être franchement défavorable.

En conclusion, et dans des conditions analogues aux nôtres, chez des poules normales $\mathrm{Dw}$, la diminution par sélection de l'un ou l'autre des deux critères de "gain de poids " envisagés ici, et surtout du premier, paraît utilisable sans inconvéniexts majeurs en vue d'améliorer dans une certaine mesure le rendement alimentaire des pondeuses; dans le cas de naines $d w$, l'intérêt d'une telle sélection est problématique.

Reçu pour publication en septembre 1976.

\section{SUMMARY}

\section{BODY WEIGHT GAIN DURING LAYING AND SELECTION ON FEED EFFICIENCY IN THE FOWL}

The body weight variation of laying hens in cages was recorded between the ages 8 and II months on hens with recorded feed consumption, between the ages $I_{7}$ weeks and Io months on other hens. Part of the hens were of the genotype Dw, part were dwarf (dw).

In the two cases, the heritability of the relevant criterion is appreciable, suggesting the possibility of improvement by breeding.

The first criterion shows among Dw hens a relatively high positive correlation with feed intake (with fixed body weight and egg mass), and a negative correlation with egg number and egg mass. The reduction by selection of this criterion of weight gain could thus improve feed efficiency of layers without unfavorable correlated reponses on other traits. The situation is comparable for dwarf hens, except the possibility of an unfavorable correlated response on average egg weight. 
For body weight variation between the ages $I_{7}$ weeks and ro months, its reduction by selection is expected to be less advantageous for Dw hens according to the observed phenotypic correlations (risk of decrease of mean egg weight) and definitely unfavorable for dwarfs (risk of decrease for egg number and mean egg weight).

\section{RÉFÉRENCES BIBLIOGRAPHIQUES}

Bordas A., Merat P., 1974. Variabilité génétique et corrélations phénotypiques caractérisant la consommation alimentaire de poules pondeuses après correction pour le poids corporel et la ponte. Ann. Génet. Sell. anim., 6, 369-379.

Byerly T. C., I94r. Feeds and other costs of producing market eggs. Univ. Maryland Agric. Exp. Sta., Bull. no A I.

LECLERCQ B., 1975. Détermination simultanée et in vivo des intensités de dépôt et de mobilisation des acides gras du tissu adipeux de la poule pondeuse. In fluence du rationnement. Ann. Biol. Anim. Bioch. Biophys., 15, 63-7I.

Leeson S., Lewis D., Shrimpton D. H., x973. Mutiple linear regression equations for the prediction of food intake in the laying fowl. Brit. Poult. Sci., 14, 595-608.

Mrrat P., rg69. Étude d'un gène de nanisme lié au sexe chez la Poule. I. Description sommaire et performances. Ann. Génét. Sél. anim., 1, I9-26.

Merat P., Ricard F. H., I974. Étude d'un gène de nanisme lié au sexe chez la Poule: Importance de l'état d'engraissement et gain de poids chez l'adulte. Ann. Génét. Sél. anim., 6, 2 II-2 I7. 